\title{
A Planar Diversity Loop Antenna Array with Improved Properties for 5G Mobile Phones
}

\author{
Naser Ojaroudi Parchin ${ }^{* 1}$, Yasir Al-Yasir' ${ }^{1}$, Mohammad Alibakhshikenari², Ahmed Maan Abdulkhaleq ${ }^{1}$, Haleh \\ Jahanabakhsh Basherlou ${ }^{3}$, Ernesto Limiti ${ }^{2}$, Jonathan Rodriguez ${ }^{4}$, and Raed A Abd-Alhameed ${ }^{1}$ \\ ${ }^{1}$ Faculty of Engineering and Informatics, University of Bradford, Bradford BD7 1DP, UK \\ ${ }^{2}$ Electronic Engineering Department, University of Rome Tor Vergata, Via del Politecnico 1, 00133 Rome, Italy \\ ${ }^{3}$ Bradford College, Bradford, West Yorkshire, BD7 1AY, UK \\ ${ }^{4}$ Instituto de Telecomunicações, Aveiro, Portugal \\ *N.OjaroudiParchin@Bradford.ac.uk
}

\begin{abstract}
A new diversity multiple-input/multiple-output (MIMO) antenna array with improved characteristics is introduced for fifth-generation (5G) mobile phone applications. Eight compact loop resonators are placed at different edges of the mobile-phone mainboard with $75 \times 150 \times 0.8 \mathrm{~mm}^{3}$ FR-4 dielectric. By addling modified arrow strips protruded among the adjacent loop antennas, the operation bandwidth and mutual coupling of the antenna radiators have been significantly improved. The MIMO antenna is designed to cover the sub $6 \mathrm{GHz}$ spectrum of 5G network and provides a wide bandwidth of 3.2-4 GHz. The MIMO performance, user-hand effect, and fundamental properties of the proposed antenna design are studied.
\end{abstract}

Keywords - 5G, cellular communications, decoupling, future mobile phones, loop radiator; MIMO.

\section{INTRODUCTION}

To support the increasing demand of high transmission rate with throughput for various fixed and mobile services, multiple-input/multiple-output (MIMO) techniques have been attracting much more attention for next-generation $5 \mathrm{G}$ communication networks [1-2]. MIMO is a promising technology to enhance the performance of $5 \mathrm{G}$ networks. In comparison with the millimeter-wave (MM-Wave) spectrum, sub $6 \mathrm{GHz}$ MIMO 5G can provide wider coverage and fewer propagation losses. In the case of massive MIMO, the $5 \mathrm{G}$ communication system also requires an array antenna with an increased number of antenna elements and dual-polarization capability [3-4]. MIMO antennas have been employed in smart and portable devices such as smartphones, laptops, etc. Among various types of MIMO antennas, microstrip antennas are more appropriate due to their attractive features. dual-polarized antennas polarization and pattern diversity could be a good candidate for use in mobile phones [5-8].

One significant drawback associated with the MIMO system is the mutual coupling between the radiation elements inside the MIMO antenna structure [9-10]. Since the space of the smart device is limited, the arrangement of the multiple antennas is difficult. Thus, the mutual coupling between the array elements is high. This means that some energy will be radiated from one element to the other one and vice versa. To maintain the radiation performance and independence of each antenna element in the MIMO system within a limited space, it is one of the urgent difficulties to overcome mutual coupling from the adjacent antenna element [11]. In addition, due to the restrictions on antenna size on mobile phone PCBs, bandwidth improvement of the MIMO antenna system without increasing the size of the antenna radiators is another challenge for smart devices. Several MIMO antennas are introduced for sub $6 \mathrm{GHz}$ 5G mobile phone applications [12-15]. However, all these antennas either offer narrow impedance bandwidth, occupy a large space, or use non-planar/single-polarized radiators.

In this work, a new bandwidth improvement and decoupling technique is introduced for radiation elements of a MIMO antenna system for $3.6 \mathrm{GHz}$ future handset applications. Eight loop antenna resonators fed by discrete ports are employed at four edge corners of the mainboard. The employed loop antennas are compact in size and printed in the same of the ground plane in the bottom layer of the substrate. By embedding an arrow strip among each loop resonator pair, the frequency bandwidth and mutual coupling characteristics are improved.

\section{Design Details of The MimO Antenna Structure}

The configuration of the MIMO mobile phone antenna is illustrated in Fig. 1. The proposed array is implemented on a single-layer FR4 dielectric with an overall size of the printed MIMO antenna is $75 \times 150 \times 1.6 \mathrm{~mm}^{3}$. The CST software is used to investigate the properties of the designed MIMO antenna [16]. Table 1 specifies the parameter values of the design.

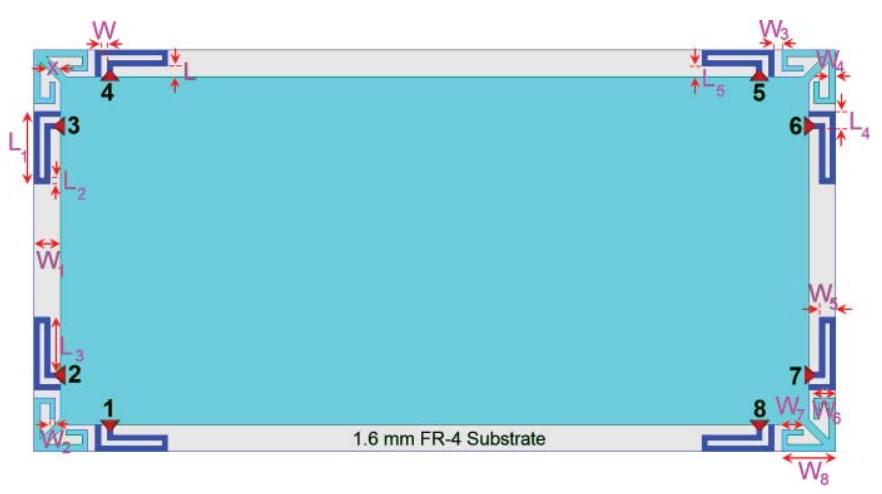

Fig. 1. The proposed 8 -element diversity mobile phone antenna. 
TABLE. I PARAMETER VALUES OF THE DESIGN

\begin{tabular}{|c|c|c|c|c|}
\hline Parameter & $\mathrm{W}$ & $\mathrm{W}_{1}$ & $\mathrm{~W}_{2}$ & $\mathrm{~W}_{3}$ \\
\hline Value (mm) & 1.25 & 5 & 1 & 1.5 \\
\hline Parameter & $\mathrm{W}_{8}$ & $\mathrm{~L}$ & $\mathrm{~L}_{1}$ & $\mathrm{~L}_{2}$ \\
\hline Value $(\mathbf{m m})$ & 10 & 2 & 13.5 & 1 \\
\hline Parameter & $\mathrm{W}_{4}$ & $\mathrm{~W}_{5}$ & $\mathrm{~W}_{6}$ & $\mathrm{~W}_{7}$ \\
\hline Value $(\mathbf{m m})$ & 1.25 & 3 & 4 & 4 \\
\hline Parameter & $\mathrm{L}_{3}$ & $\mathrm{~L}_{4}$ & $\mathrm{~L}_{5}$ & $\mathrm{X}$ \\
\hline Value $(\mathbf{m m})$ & 10.3 & 3.2 & 2 & 1.5 \\
\hline
\end{tabular}

\section{Characteristics of The Proposed Antenna ArRay}

To explain the bandwidth enhanced and reduced mutual coupling characteristic, we investigated the performance of the antenna pairs without and with the decoupling structure. Figure 2 shows the $S_{11} / S_{21}$ results of the adjacent loop elements with/without the modified arrow strip. As shown, the loop resonator is operating at $3.6 \mathrm{GHz}$ fulfilled the requirement of sub $6 \mathrm{GHz}$ cellular communication. In addition, the $\mathrm{S}_{21}$ of the basic design is around $-10 \mathrm{~dB}$. It is evident that by embedding a decoupling strip among the closely-spaced loop resonators, the frequency bandwidth and mutual coupling characteristics are improved. As seen, another resonance is also generated at the lower band to improve the impedance bandwidth of the design. Therefore, $800 \mathrm{MHz}$ impedance bandwidth in the frequency spectrum of $3.2-4 \mathrm{GHz}$ is obtained for the proposed $5 \mathrm{G}$ antenna. Nevertheless, for $\mathrm{S}_{11} \leq-6 \mathrm{~dB}$, the loop antenna element is supporting the operation band of $3-4.3 \mathrm{GHz}$. On the other hand, the result of $\mathrm{S}_{21}$ is better than $-12 \mathrm{~dB}$ and it has reached $-22 \mathrm{~dB}$ at the middle frequencies of the antenna operation band.

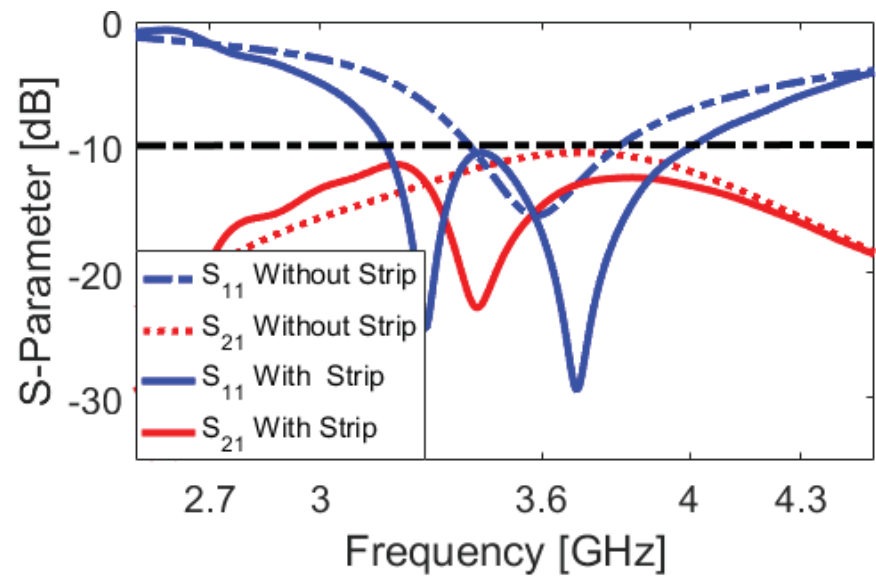

Fig. 2. $\mathrm{S}_{11} / \mathrm{S}_{21}$ of adjacent elements with/without the modified decoupling strip.

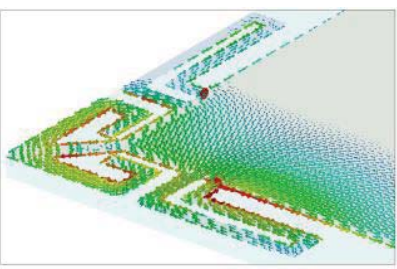

(a)

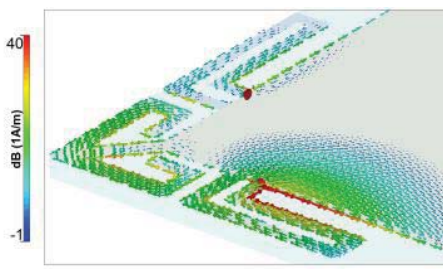

(b)
Fig. 3. Simulated current densities at (a) $3.3 \mathrm{GHz}$ and (b) $3.7 \mathrm{GHz}$.
To validate the effectiveness of the employed strip among the antenna pairs, the current densities of the antenna element at $3.3 \mathrm{GHz}$ and $3.7 \mathrm{GHz}$ (resonant frequencies) are investigated in Fig. 3. It is seen that at the first resonance $(3.3 \mathrm{GHz})$, the surface current is distributed around the employed decoupling arrow strip verifying its role in generating a new resonance at the lower band which leads to increase the antenna bandwidth [17-18]. While at the second resonance, the currents have been concentrated inside the loop resonator.

Figures 4 (a) and (b) illustrate $\mathrm{S}_{\mathrm{nn}}$ (reflection coefficient) and $\mathrm{S}_{\mathrm{mn}}$ (transmission coefficient) results of the designed array, respectively. As seen from Fig. 4 (a), a wide operating frequency band of $800 \mathrm{MHz}$ for $\mathrm{S}_{\mathrm{nn}} \leq-10$ and $1300 \mathrm{MHz}$ for $\mathrm{S}_{\mathrm{nn}} \leq-6 \mathrm{~dB}$ are obtained. Sufficient mutual couplings are observed for the antenna elements, as illustrated in Fig. 4 (b).

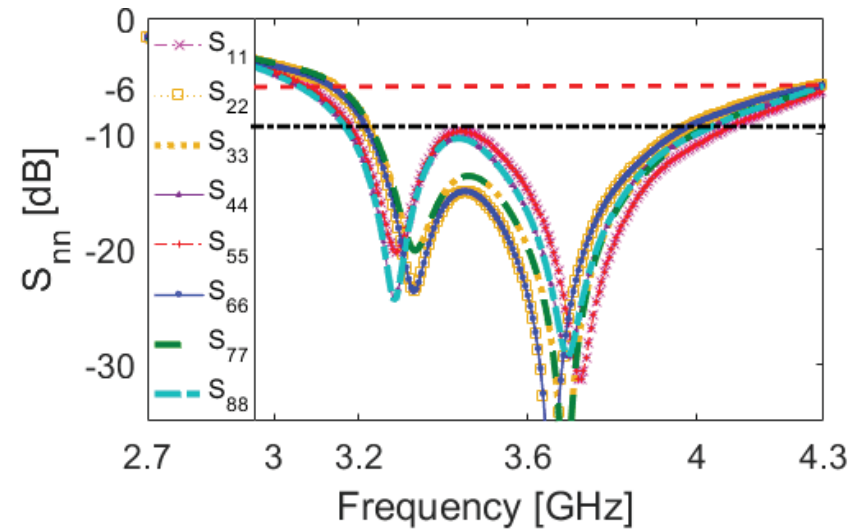

(a)

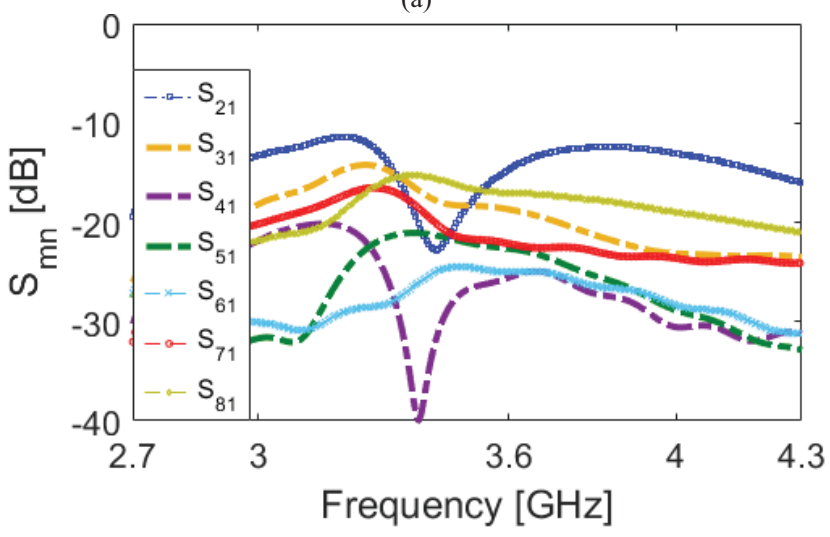

(b)

Fig. 4. Simulated (a) $\mathrm{S}_{\mathrm{nn}}$ and (b) $\mathrm{S}_{\mathrm{mn}}$ results of the studied array.

The efficiencies (radiation and total) of the MIMO antenna system are also depicted in Fig. 5. It is shown that sufficient efficiency results are obtained within the operation bandwidth of the design: at $3.4-4 \mathrm{GHz}$, more than $80 \%$ radiation and $60 \%$ total efficiencies are achived for the proposed smartphone atenna. The three-dimensional variation of the radiated field for the elements are studied at the middle frequency and represented in Fig. 6. This figure gives more appreciation of the field shape and shows the elements of the MIMO antenna are capable to support different sides of handset and improve the performance of the design [19-20]. In addition, good gain values are achieved for each loop antenna resonator. 


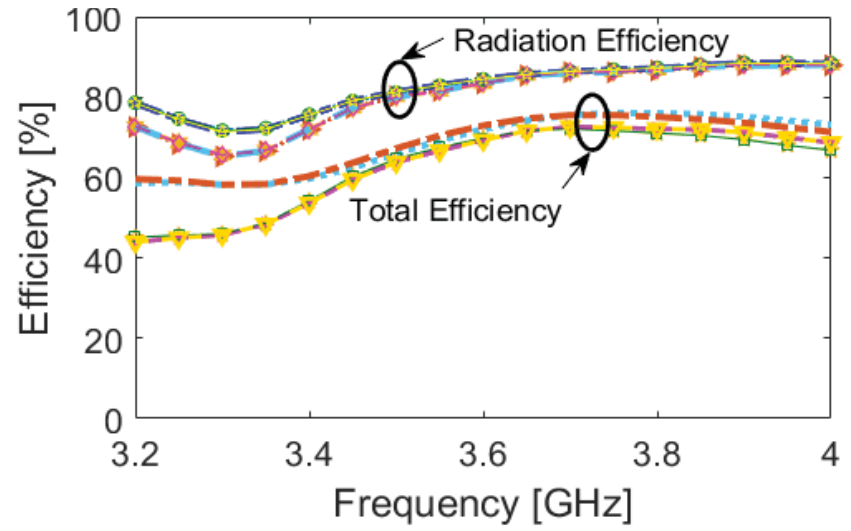

Fig. 5. Efficiencies of the MIMO diversity mobile-phone antenna.

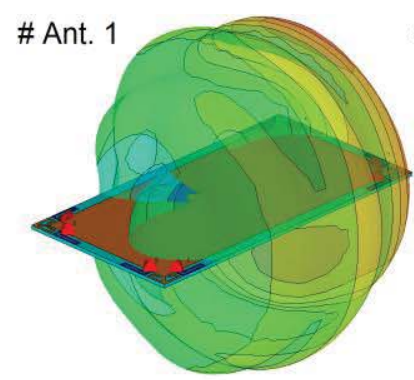

\# Ant. 3

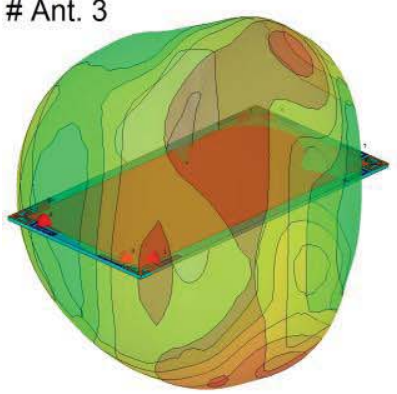

\# Ant. 5

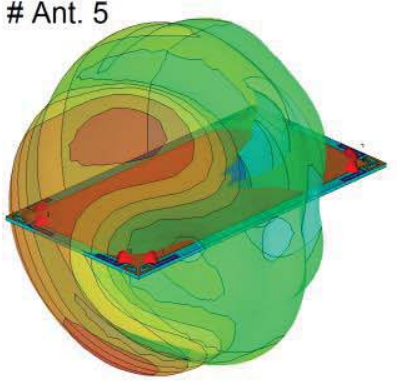

\# Ant. 7

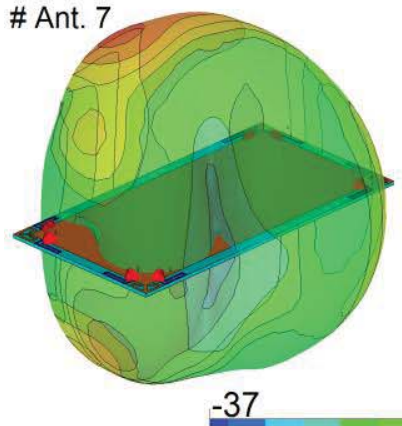

\# Ant. 8

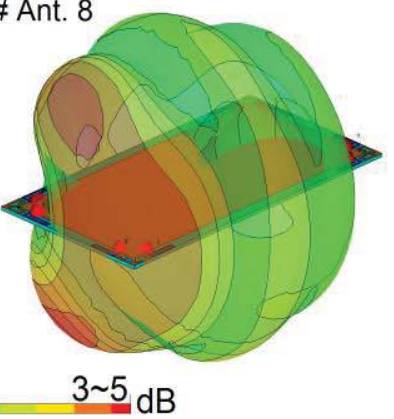

Fig. 6. Radiation patterns of diversity loop element at $3.6 \mathrm{GHz}$.
As illustrated in Fig. 7 (a), the 5G smartphone antenna was fabricated and the S-parameters of the adjacent loop resonators are measured and discussed. The feeding mechanism of the adjacent loop antenna elements is also depicted in Fig. 7 (b). Figures 8 (a) and (b) compares the $\mathrm{S}_{11} / \mathrm{S}_{21}$ and envelope correlation coefficient (ECC) properties of the simulation and measurements. The practical results show fair agreements with the simulated outcomes. for $\mathrm{S}_{11} \leq-10 \mathrm{~dB}$, the loop element is supporting the operation band of $3.2-4 \mathrm{GHz}$ and achieving quite good mutual coupling.

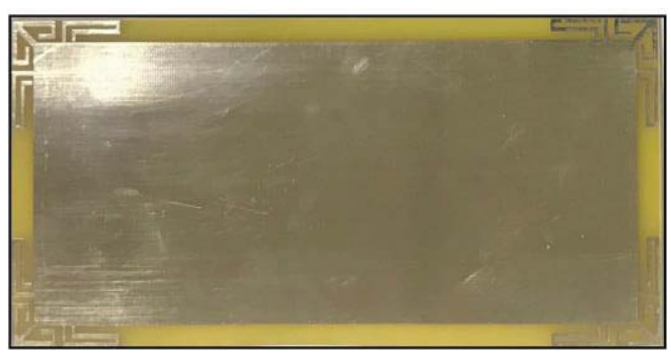

(a)

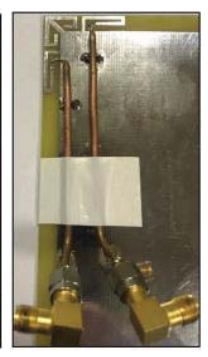

(b)
Fig. 7. (a) Prototype sample and (b) feeding mechanism.

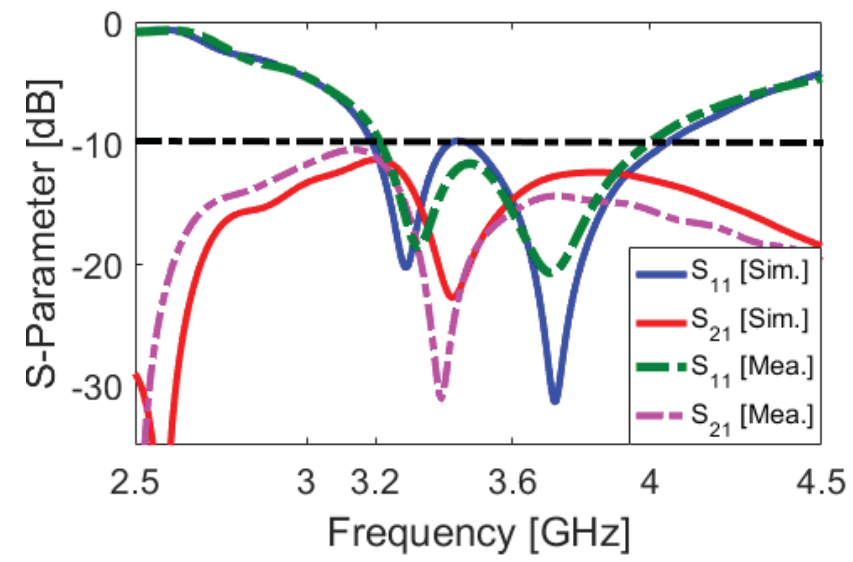

(a)

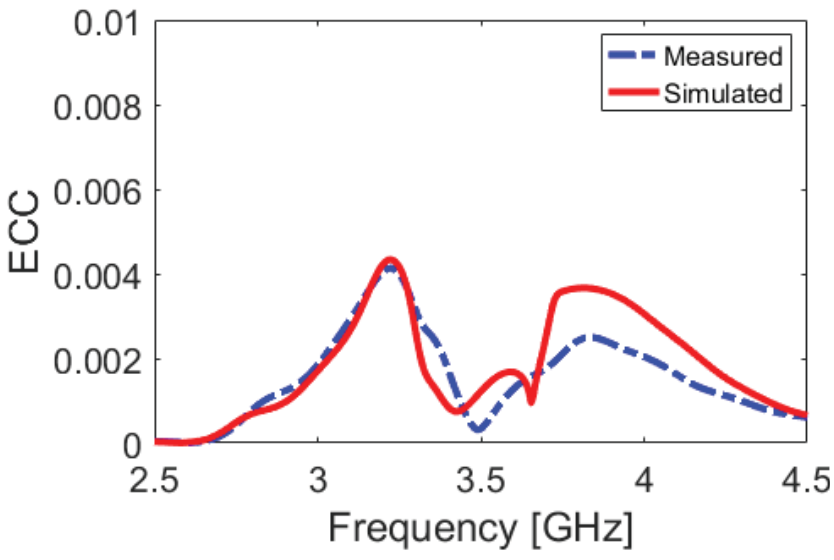

(b)

Fig. 8. Measured and simulated (a) $\mathrm{S}_{11} / \mathrm{S}_{21}$ and (b) ECC results.

The impact of user-hand on the efficiency of the MIMO design for different usage postures are discussed in Fig. 9. Due to the symmetrical schematic of the design, the user-effect for different studied scenarios is almost similar and good 
efficiency results (20\%-60\%) are achieved. It worth noting that the maximum effects are observed for the element partially covered by hand phantom [21-22].
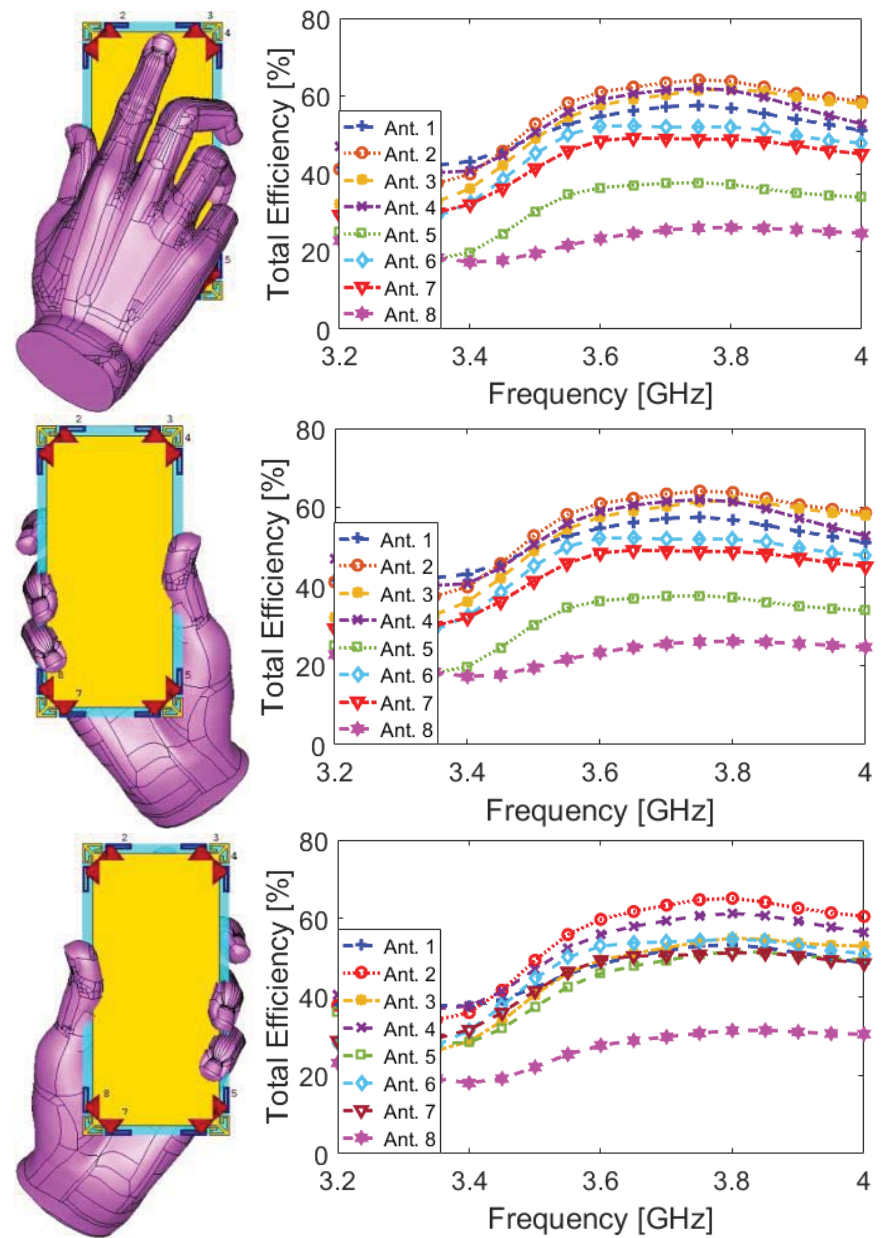

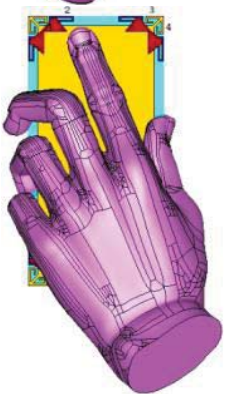

(a)

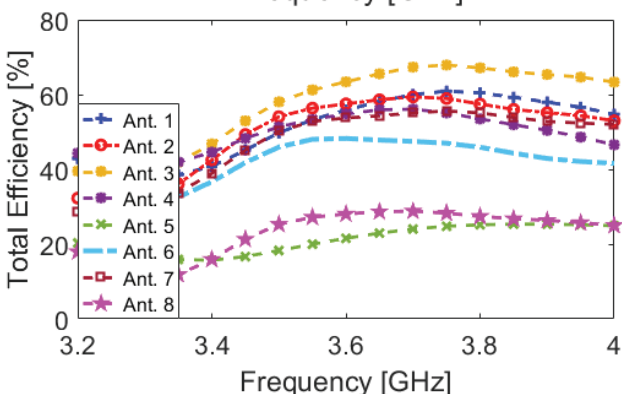

(b)
Fig. 9. (a) The placement and (b) efficiency results of the proposed mobile phone antenna in the user-hand investigation.

\section{CONCLUSION}

A new planar loop antenna array with improved properties is proposed for $5 \mathrm{G}$ mobile phone applications. By embedding an arrow strip among each loop resonator pair, the frequency bandwidth and mutual coupling characteristics are improved. The S-parameters and radiation characteristics of the proposed MIMO antenna system are discussed, and good results are achieved.

\section{ACKNOWLEDGMENT}

This work is supported by the European Union's Horizon 2020 research and innovation programme under grant agreement H2020-MSCA-ITN-2016 SECRET-722424.

\section{REFERENCES}

[1] A. Osseiran, et al., "Scenarios for 5G mobile and wireless communications: the vision of the METIS project," IEEE Commun. Mag., vol. 52, pp.26-35, 2014.

[2] M. Jensen, J. Wallace, "A review of antennas and propagation for MIMO wireless communications", IEEE Trans. Antennas Propag., vol. 52, 2810-2824, 2004.

[3] J. Rodriguez, et al. "SECRET-Secure Network Coding for Reduced Energy next Generation Mobile Small Cells: A European Training Network in Wireless Communications and Networking for 5G." Internet Technologies and Applications (ITA), pp. 329-333. 2017.

[4] Q.U.A. Nadeem, et al., "Design of 5G full dimension massive MIMO systems," IEEE Trans. Commun., vol. 66, pp. 726-740, 2018.

[5] M. S. Sharawi, "Printed MIMO antenna engineering," 2014, Norwood, MA, USA: Artech House.

[6] M.S. Sharawi, "Printed multi-band MIMO antenna systems and their performance metrics [wireless corner]," IEEE Antennas Propag. Mag., Vol. 55, 218-232, 2013

[7] H.H. Yang,; Y.Q.S. Quel, "Massive MIMO Meet Small Cell. SpringerBriefs in Electrical and Computer Engineering, 2017. DOI 10.1007/978-3-319-43715-6 2.

[8] N. Ojaroudi, N. Ghadimi, "Design of CPW-Fed slot antenna for MIMO system applications," Microwave Opt Technol Lett vol. 56, pp.12781281,2014

[9] M. Alibakhshikenari, et al., "Mutual-coupling isolation using embedded metamaterial EM bandgap decoupling slab for densely packed array antennas," IEEE Access, vol. 7, pp. 5182-51840, 2019.

[10] M. Alibakhshikenari, et al., "Meta-surface wall suppression of mutual coupling between microstrip patch antenna arrays for THz-band applications", Progress In Electromagnetics Research Letters, vol. 75, pp. 105-111, 2018.

[11] N. O. Parchin, et al., "A closely spaced dual-band MIMO patch antenna with reduced mutual coupling for 4G/5G applications," Progress In Electromagnetics Research C, vol. 101, pp. 71-80, 2020.

[12] M.-Y. Li, et al., "Tri-polarized 12-antenna MIMO array for future 5G smartphone applications," IEEE Access, vol. 6, pp. 6160-6170, 2018.

[13] N. O. Parchin, et al., "Dual-polarized MIMO antenna array design using miniaturized self-complementary structures for $5 \mathrm{G}$ smartphone applications," EuCAP Conference, April 2019, Krakow, Poland.

[14] A. Zhao, R. Zhouyou, "Size reduction of self-isolated MIMO antenna system for 5G mobile phone applications," IEEE Antennas and Wireless Propagation Letters, vol. 18, pp. 152-156, 2019.

[15] Y. Li, et al., "High-isolation 3.5-GHz 8-antenna MIMO array using balanced open slot antenna element for 5G smartphones," IEEE Trans. Antennas Propag., vol. 67, pp. 3820-3830, 2019.

[16] CST Microwave Studio, ver. 2017, CST, Framingham, MA, USA, 2017.

[17] A. Kamalvand, et al., "Omnidirectional/multi-resonance CPW-fed small slot antenna for UWB applications," ACES Journal, vol. 28, pp. 829-835, 2013.

[18] A. Musavand, et al., "A compact UWB slot antenna with reconfigurable band-notched function for multimode applications," ACES Journal, vol. 31, pp. 14-18, 2016

[19] Y.I.A. Al-Yasir, et al., "A new polarization-reconfigurable antenna for 5G applications," Electronics, vol. 7, pp. 1-9, 2018.

[20] N. O. Parchin et al., "Frequency reconfigurable antenna array with compact end-fire radiators for $4 \mathrm{G} / 5 \mathrm{G}$ mobile handsets," IEEE 2 nd $5 G$ World Forum (5GWF), Dresden, Germany, 2019.

[21] I. Syrytsin, S. Zhang, G. F. Pedersen, "Performance investigation of a mobile terminal phased array with user effects at $3.5 \mathrm{GHz}$ for LTE advanced," IEEE Antennas Wirel. Propag. Lett., vol. 16, pp. 18471850, 2017.

[22] R. Khan, et al., User influence on mobile terminal antennas: A review of challenges and potential solution for 5G antennas, IEEE Access, vol. 6, pp. 77695-77715, 2018. 